Prahlad K. Sethi

Nitin K. Sethi

Josh Torgovnick

\section{Red forehead dot syndrome and migraine}

Received: 9 January 2007

Accepted in revised form: 23 January 2007

Published online: 11 May 2007
Dear Editor,

Migraine headaches are recurrent unilateral or bilateral headaches commonly associated with other symptoms like nausea, dizziness and complaints of photophobia and phonophobia. Autonomic nervous system dysfunction has been reported in migraine and orthostatic hypotension has been reported to be more common in migraineurs [1]. Other symptoms like red ear syndrome (RES) have been reported to be causally related to activation of the trigeminovascular system.

We present here a patient with migraine, who at the time of his migraine attack used to notice a red dot on his forehead. The appearance of this red dot had a temporal relationship to his headaches. A 40-yearold right-hand-dominant male presented to us for evaluation of his headaches. He recounted having headaches since the age of 16 . Headaches were described as episodic, throbbing in character, three to four times a month, mostly on the weekends. They were hemicranial, more frequently on the left but also on the right, and associated with nausea. He had vomited twice during his headache episodes.

When he changed his job and moved to Riyadh (Saudi Arabia) he noticed that the character of his headache changed. Now instead of getting his headaches on Saturday and Sunday, he started getting them on Thursday and Friday, which is the weekend in Saudi Arabia. At that time he decided to work on the weekends too, so as to escape his headaches.

He denied any preceding aura of visual scintillation but had noticed the appearance of a red dot on his forehead at the time of his headaches (Fig. 1). Appearance of this red dot had a temporal relationship to his headaches with its appearance at the onset and disappearance at the termination of an attack. Neurological examination, magnetic resonance imaging (MRI) of the brain and Xrays of the cervical spine were normal.

Migraine pathophysiology involves a complex interaction leading to trigeminovascular activation. Thus migraine pain is referred to the trigeminal distribution as well as beyond the trigeminal domain, such as neck pain, due to the overlap between the neurons of the trigeminal nucleus caudalis and neurons located within the $\mathrm{C} 1$ and $\mathrm{C} 2$ dorsal horns of the upper cervical cord. RES has been previously described in the literature and its temporal correlation to migraine has been well documented [2]. Primary or secondary 


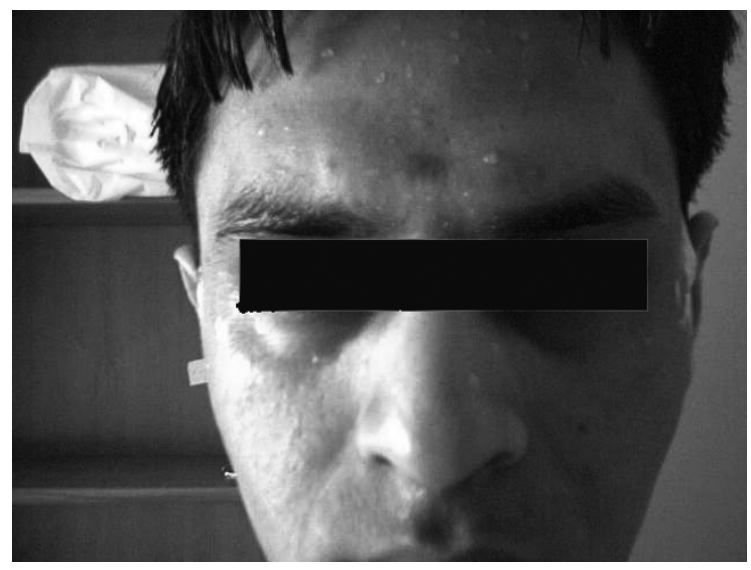

Fig. 1 Photograph of the patient showing red forehead dot sign, with redness extending in the $\mathrm{V} 1$ distribution of the trigeminal nerve. Profuse sweating is also noticed during one of his severe attacks of migraine

trigeminovascular activation leading to extracerebral vasodilatation via the release of nitric oxide, substance $\mathrm{P}$ or other mediators may lead to RES as well as the red forehead dot seen in our patient.

On closer inspection our patient did not only have a red dot; the redness extended to the periorbital area in the classic V1 distribution of the trigeminal nerve. On further questioning he volunteered that the red dot appeared in about $20 \%$ of attacks, more commonly the severe ones, and was associated with profuse sweating. To the best of our knowledge, this is the first documented pictorial evidence of this intriguing and fascinat- ing clinical sign associated with migraine.

P.K. Sethi

Department of Neurology, Sir Ganga Ram Hospital, New Delhi, India

N.K. Sethi $(Y)$

Comprehensive Epilepsy Center, Weill Cornell Medical Center, New York, NY 10021, USA e-mail: sethinitinmd@hotmail.com Tel.: +1-646-5155168 Fax: +1-212-7466684

J. Torgovnick Department of Neurology, Saint Vincent's Hospital and Medical Centers, New York, NY, USA

\section{References}

1. Peroutka SJ (2004) Migraine: a chronic sympathetic nervous system disorder. Headache 44:53-64
2. Raieli V, Monastero R, Santangelo G et al (2002) Red ear syndrome and migraine: a report of 8 cases. Headache 42:147-151 